\title{
Two-loop five-point amplitudes in QCD from Feynman diagrams
}

\section{Heribertus Bayu Hartanto*}

Institute for Particle Physics Phenomenology, Department of Physics, Durham University, Durham DH1 3LE, United Kingdom

E-mail: heribertus.b.hartanto@durham.ac.uk

\begin{abstract}
We present an approach to compute two-loop amplitudes combining Feynman diagram input, numerical sampling over finite fields and functional reconstruction techniques. We apply the method to compute numerically $W+4$ parton scattering amplitudes and to derive analytical form of four-quark one-gluon amplitudes, both in the leading colour approximations.
\end{abstract}

European Physical Society Conference on High Energy Physics - EPS-HEP2019

10-17 July, 2019

Ghent, Belgium

${ }^{*}$ Speaker. 


\section{Introduction}

Increasing the precision of theoretical predictions to assist LHC experiments, requires the knowledge of multi-loop scattering amplitudes. With the theoretical predictions accurate at nextto-next-to leading order (NNLO) in QCD has become new standard in the recent years, the knowledge of two loop scattering amplitudes becomes more and more important. Many processes that are essential for LHC phenomenology are of multiscale nature (those with many legs or masses present). In dealing with multiscale scattering amplitudes, we potentially encounter algebraic and analytical challenges. The past few years have seen tremendous efforts in the attempt to attack the algebraic challenge, using the combination of numerical computation over finite fields, and analytical reconstruction techniques [1]. Many important results for massless 2 to 3 scattering amplitudes with five external partons have been derived using such an approach, together with differential equation techniques to solve Feynman integrals [2-13]. In the computation of planar two-loop five parton amplitudes, tree-level amplitudes have been used to construct the integrand via generalised unitarity cuts. In this proceeding, we demonstrate the use of Feynman diagrams as numerator input in the integrand construction, in conjunction with numerical computation over finite-fields and analytic reconstruction on the final results. We apply this method to compute the planar $W+4$ parton scattering amplitudes numerically and derive the analytic expressions for the leading colour four-quark one-gluon amplitudes.

\section{Computational setup}

We generate a set of Feynman diagram using QGRAF [14] and perform colour decomposition to obtain the colour-ordered amplitude. The numerator of Feynman diagrams that share the same topology are grouped into a common numerator topology $N_{T}$. After fixing the helicity of external particles and performing t'Hooft algebra with the help of SPINNEY [15] library, we obtained the following form of colour-ordered amplitude

$$
A_{n}^{(2), h}(\{p\})=\int \prod_{i=1}^{2} \frac{d^{d} k_{i}}{i \pi^{d / 2} e^{-\varepsilon \gamma_{E}}} \sum_{T} \frac{N_{T}^{h}\left(d_{s},\{k\},\{p\}\right)}{\prod_{\alpha \in T} D_{\alpha}(\{k\},\{p\})},
$$

where $k_{i}$ is the loop momenta, $p_{i}$ is the external momenta, $d=4-2 \varepsilon$ is the space-time dimension, $d_{s}=g_{\mu}^{\mu}$ is the spin dimension, and $D_{\alpha}(\{k\},\{p\})$ is a set of loop propagator denominators for each diagram topology $T$. We decompose the $d$-dimensional loop momentum into its 4- and extradimensional components, $k=\bar{k}+\tilde{k}$. In order to evaluate the external kinematics over finite fields, we require rational phase-space parametrisation that can be achieved through the use of momentum twistor variables $x_{i}$ [16]. The explicit functional dependence of the helicity-dependent numerator topology $N_{T}^{h}$ is

$$
N_{T}^{h}\left(d_{s},\{k\},\{p\}\right)=N_{T}^{h}\left(d_{s}, x_{i}, k_{i} \cdot k_{j}, \bar{k}_{i} \cdot p_{j}, \mu_{i j},\left\langle p_{a}\left|\bar{k}_{i}\right| p_{b}\right],\left\langle p_{a}\left|\bar{k}_{i}\right| \bar{k}_{j} \mid p_{b}\right\rangle,\left[p_{a}\left|\bar{k}_{i}\right| \bar{k}_{j} \mid p_{b}\right]\right),
$$

where $\mu_{i j}=\tilde{k}_{i} \cdot \tilde{k}_{j}$. We apply integrand reduction algorithm to construct irreducible numerators for each topology $\Delta_{T}^{h}$,

$$
A_{n}^{(2), h}(\{p\})=\int \prod_{i=1}^{2} \frac{d^{d} k_{i}}{i \pi^{d / 2} e^{-\varepsilon \gamma_{E}}} \sum_{T} \frac{\Delta_{T}^{h}\left(d_{s},\{k\},\{p\}\right)}{\prod_{\alpha \in T} D_{\alpha}(\{k\},\{p\})} .
$$


$\Delta_{T}^{h}$ is made up of a basis of irreducible scalar products (ISPs) of topology $T, \Delta_{T}=\sum c_{i}^{T} m_{T}$ (ISP). We determine the coefficients of monomial basis of ISPs for each topology $c_{i}^{T}$ using OPP-style top-down approach [17]. There are freedom in choosing what type of ISP basis to be used. ISP basis containing extra-dimensional component of the loop momentum $\left(k \cdot p_{i}, \mu_{i j}\right)$ can be utilised to obtain compact integrand representations. In this work we use auxiliary propagators as our ISP basis, that is directly compatible with integration-by-parts (IBP) reduction. In order to fix $c_{i}^{T}$, we first need to express the loop momentum dependent objects in Eq. 2.2 in terms of propagators and ISPs. While objects like $k_{i} \cdot k_{j}$ and $\bar{k}_{i} \cdot p_{j}$ can easily be written as propagators and ISPs, objects like $\left\langle p_{a}\left|\bar{k}_{i}\right| p_{b}\right],\left\langle p_{a}\left|\bar{k}_{i}\right| \bar{k}_{j} \mid p_{b}\right\rangle$ and $\left[p_{a}\left|\bar{k}_{i}\right| \bar{k}_{j} \mid p_{b}\right]$ require special treatment. We parametrise the 4dimensional component of the loop momenta in terms of spanning vectors $v$,

$$
\bar{k}_{i}^{\mu}=\sum_{j=1}^{4} a_{i j} \text { (propagators, ISPs) } v_{j}^{\mu} .
$$

For topologies with five or more external legs, $v^{\mu}$ consists only of external momenta, while for topologies with four external legs or fewer, spurious vectors need to be included in $v^{\mu}$. Once the integrand representation of the helicity amplitude is constructed, we can further perform IBP reduction to write the amplitude in a basis of master integral $\operatorname{MI}_{k}(\{p\}, \varepsilon)$

$$
A_{n}^{(2), h}(\{p\})=\sum_{k} c_{k}^{\mathrm{IBP}}\left(x_{i}, \varepsilon\right) M I_{k}(\{p\}, \varepsilon) .
$$

IBP identities are generated in MATHEMATICA using LiteRed [18], and solved using Laporta approach [19]. Note that in our setup, the whole chain of computation starting from Feynman diagram numerators down to the master integral representation of the amplitude in Eq. 2.5, is performed numerically over finite fields within the FINITEFLOW framework [20]. In the processes with low multiplicity $\left(2 \rightarrow 2\right.$ or lower), typically the analytic form of $c_{k}^{\mathrm{IBP}}\left(x_{i}, \varepsilon\right)$ can be reconstructed from several numerical evaluations. In the higher multiplicity case, however, the polynomials in $c_{k}^{\mathrm{IBP}}\left(x_{i}, \varepsilon\right)$ are of high degree thus the evaluation and reconstruction become very computationally demanding. In the case where the analytic solution of master integrals are known, and the expansion to special function basis is possible, we can subtract the singular part of the two-loop amplitude, and analytically reconstruct only the finite remainder. The polynomial complexity of the finite remainder typically are much lower compared to the amplitude in the master integral basis.

\section{A numerical evaluation of planar two-loop $W+4$ parton helicity amplitudes}

We apply the computational framework discussed in the previous section to compute twoloop $W+4$ parton scattering amplitudes in the leading colour approximation, including the $W \rightarrow$ $\bar{v} \ell$ decay. We consider both the $q \bar{Q} Q \bar{q}^{\prime} \bar{v} \ell$ and $q g g \bar{q}^{\prime} \bar{v} \ell$ subprocesses, with the following colour decompositions

$$
\begin{aligned}
& \mathscr{A}^{(L)}\left(1_{q}, 2_{\bar{Q}}, 3_{Q}, 4_{\bar{q}^{\prime}}, 5_{\bar{v}}, 6_{\ell}\right)=n^{L} g_{s}^{2} g_{W}^{2} \delta_{i_{1}}^{\bar{i}_{2}} \delta_{i_{3}}^{\bar{i}_{4}} A^{(L)}\left(1_{q}, 2_{\bar{Q}}, 3_{Q}, 4_{\bar{q}^{\prime}}, 5_{\bar{v}}, 6_{\ell}\right), \\
& \mathscr{A}^{(L)}\left(1_{q}, 2_{g}, 3_{g}, 4_{\bar{q}^{\prime}}, 5_{\bar{v}}, 6_{\ell}\right)=n^{L} g_{s}^{2} g_{W}^{2}\left[\left(T^{a_{2}} T^{a_{3}}\right)_{i_{1}}^{\bar{i}_{4}} A^{(L)}\left(1_{q}, 2_{g}, 3_{g}, 4_{\bar{q}^{\prime}}, 5_{\bar{v}}, 6_{\ell}\right)+(2 \leftrightarrow 3)\right],
\end{aligned}
$$


where $n=m_{\varepsilon} \alpha_{s} /(4 \pi)$ is the overall normalisation, $\alpha_{s}=g_{s}^{2} /(4 \pi)$ and $m_{\varepsilon}=i(4 \pi)^{\varepsilon} e^{-\varepsilon \gamma_{E}}$. The first step towards deriving analytic results for $W+4$ parton planar scattering amplitudes is to perform numerical benchmarking at a specific phase-space point. We use momentum twistor parametrization for massless kinematics with 6 external particles, that involves 8 kinematic variables. Master integrals appearing in the planar $W+4$ parton scattering are known analytically for the case of four external legs or fewer [21-26]. For master integrals with five external legs, only one of the planar family has been computed [27]. The missing master integrals are computed numerically using FIESTA [28] and PYSECDEC [29]. It is challenging, however, to obtain numerical results with decent accuracies, since large cancellations occur in the amplitude. To alleviate this issue, we introduce a basis of master integral with local numerator insertions for the missing master integrals. Such basis of master integral possesses improved singularity behaviour that is beneficial in the evaluation using sector decomposition method. For example, the standard master integrals for one of the pentagon-box topology are

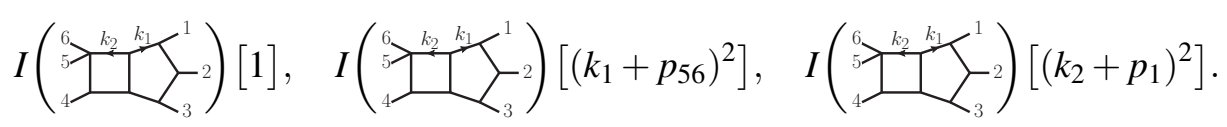

The Laurent expansion of these integrals starts at $\mathscr{O}\left(\varepsilon^{-4}\right), \mathscr{O}\left(\varepsilon^{-3}\right)$ and $\mathscr{O}\left(\varepsilon^{-3}\right)$ respectively. The master integrals with local numerator insertion for this topology are

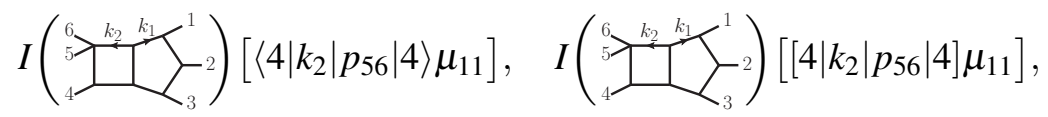

$$
\begin{aligned}
& I\left({ }_{5}^{6} \int_{4}^{k_{2}} \sum_{-1}^{k_{1}} \sum_{-2}^{1}\right)\left[\operatorname{tr}_{-}\left(1\left(k_{1}-p_{1}\right)\left(k_{1}-p_{12}\right) 3\right)\left\langle 4\left|k_{2}\right| p_{56} \mid 4\right\rangle\right],
\end{aligned}
$$

where $\operatorname{tr}_{ \pm}(i j k l)=\frac{1}{2} \operatorname{tr}\left(\left(1 \pm \gamma_{5}\right) \not p_{i} \not_{j} \not_{k} \phi_{l}\right)$. These local master integrals evaluate to $\mathscr{O}(\varepsilon), \mathscr{O}(\varepsilon)$ and $\mathscr{O}(1)$ respectively.

In Table 1, we present numerical results for $q \bar{Q} Q \bar{q}^{\prime} \bar{v} \ell$ and $q g g \bar{q}^{\prime} \bar{v} \ell$ processes in the t'HooftVeltman (HV) scheme for each independent helicity configurations using the following Euclidean phase-space point

$$
\begin{gathered}
x_{1}=-1, \quad x_{2}=\frac{79}{270}, \quad x_{3}=\frac{64}{61}, \quad x_{4}=-\frac{37}{78}, \quad x_{5}=\frac{83}{102}, \\
x_{6}=\frac{4723}{9207}, \quad x_{7}=-\frac{12086}{7451}, \quad x_{8}=\frac{3226}{2287},
\end{gathered}
$$

normalised to the tree level amplitude according to

$$
\hat{A}_{\lambda_{1} \lambda_{2} \lambda_{3} \lambda_{4} \lambda_{5} \lambda_{6}}^{(2)}=\frac{A^{(2)}\left(1^{\lambda_{1}}, 2^{\lambda_{2}}, 3^{\lambda_{3}}, 4^{\lambda_{4}}, 5^{\lambda_{5}}, 6^{\lambda_{6}}\right)}{A^{(0)}\left(1^{\lambda_{1}}, 2^{\lambda_{2}}, 3^{\lambda_{3}}, 4^{\lambda_{4}}, 5^{\lambda_{5}}, 6^{\lambda_{6}}\right)} .
$$

We have verified that the divergent part of the amplitudes presented in Table 1 agree with universal singularity structures at two loops [30-33] in the HV scheme.

\section{Analytic form of planar 4-quark 1-gluon amplitudes}

In this section, we present a computation of $q \bar{q} Q \bar{Q} g$ two-loop amplitudes in QCD in the leading colour approximation, which is an independent calculation of [8]. In the planar massless two-loop 


\begin{tabular}{|l|c|c|c|c|c|}
\hline \multicolumn{1}{|c|}{$q g g \bar{q}^{\prime} \bar{v} \ell$} & $\varepsilon^{-4}$ & $\varepsilon^{-3}$ & $\varepsilon^{-2}$ & $\varepsilon^{-1}$ & $\varepsilon^{0}$ \\
\hline$\widehat{A}_{-+++++-}^{(2)}$ & 4.50000 & $-3.63577(3)$ & $-277.2182(7)$ & $-344.56(1)$ & $2051.1(2)$ \\
$\widehat{A}_{-+-++-}^{(2)}$ & 4.50000 & $-3.63581(9)$ & $-13.6826(2)$ & $6.143(5)$ & $66.21(7)$ \\
$\widehat{A}_{--+++-}^{(2)}$ & 4.50000 & $-3.63579(5)$ & $-18.79219(7)$ & $-6.633(6)$ & $79.02(4)$ \\
\hline$q \bar{Q} Q \bar{q}^{\prime} \bar{v} \ell$ & $\varepsilon^{-4}$ & $\varepsilon^{-3}$ & $\varepsilon^{-2}$ & $\varepsilon^{-1}$ & $\varepsilon^{0}$ \\
\hline$\widehat{A}_{-+-+++}^{(2)}$ & 2.00000 & $-7.16949(9)$ & $-9.9055(2)$ & $39.922(6)$ & $154.79(7)$ \\
$\widehat{A}_{--+++-}^{(2)}$ & 2.00000 & $-7.16948(8)$ & $-12.9371(1)$ & $41.432(8)$ & $189.53(6)$ \\
\hline
\end{tabular}

Table 1: Numerical results for $q \bar{Q} Q \bar{q} \bar{v} \ell$ and $q g g \bar{q} \bar{v} \ell$ helicity amplitudes in the leading colour limit, using kinematic points defined in Eq. (3.5), in the HV scheme.

five-point case, the analytic solutions of the master integrals are known in the basis of the so-called pentagon functions [34]. We map the master integrals $M I_{k}$ in Eq. (2.5) into a combination of pentagon function monomials $m_{l}(f)$ and subtract the divergent part of the amplitude followed by Laurent expansion in $\varepsilon$ to obtain the two-loop finite remainder

$$
F_{n}^{(2), h}(\{p\})=\sum_{l} c_{l}^{\mathrm{F}, h}\left(x_{i}\right) m_{l}(f(\{p\}))+\mathscr{O}(\varepsilon)
$$

The coefficients $c_{l}^{\mathrm{F}, h}\left(x_{i}\right)$ appearing in (4.1), however, are not all independent. We exploit this fact in order to simplify both the result and the reconstruction of its analytic expression, as follows. First, we sort all the coefficients by their complexity, which is estimated from their total degree. The total degree, in turn, can be quickly determined via an univariate fit, as explained in ref. [1]. We then find vanishing linear combinations of these coefficients by solving the linear fit problem

$$
\sum_{l} y_{l} c_{l}^{\mathrm{F}, h}\left(x_{i}\right)=0
$$

with respect to the unknowns $y_{l}$. This allows us to find linear relations between the coefficients, which rewrite the more complicated ones in terms of the simpler ones. After applying the linear relations between coefficients of pentagon function monomials, we have

$$
F_{n}^{(2), h}(\{p\})=\sum_{l} \bar{c}_{l}^{\mathrm{F}, h}\left(x_{i}\right) \bar{m}_{l}(f(\{p\}))+\mathscr{O}(\varepsilon)
$$

where $\bar{c}_{l}^{\mathrm{F}}$ are the independent coefficients of pentagon function monomials and $\bar{m}_{l}(f)$ are new sets of pentagon function monomials that are linear combinations of monomials appearing in Eq. (4.1). Therefore, functional reconstruction only needs to be applied to the independent coefficients $\bar{c}_{l}^{\mathrm{F}}$. This yields a significantly simpler result than the one in Eq. (4.1), and also reduces the number of evaluations needed for its reconstruction.

The colour decomposition of unrenormalized $q \bar{q} Q \bar{Q} g$ amplitudes at leading colour is given by

$$
\mathscr{A}^{(L)}\left(1_{q}, 2_{\bar{q}}, 3_{Q}, 4_{\bar{Q}}, 5_{g}\right)=n^{L} g_{s}^{3}\left[\left(T^{a_{5}}\right)_{i_{1}}^{\bar{i}_{4}} \delta_{i_{3}}^{\bar{i}_{2}} A^{(L)}\left(1_{q}, 2_{\bar{q}}, 3_{Q}, 4_{\bar{Q}}, 5_{g}\right)+(1 \leftrightarrow 3,2 \leftrightarrow 4)\right] .
$$


The finite remainder of $L$-loop amplitude is obtained by subtracting the infrared (IR) and ultraviolet (UV) poles from the unrenormalized colour-ordered amplitude

$$
\mathscr{F}^{(L)}\left(1_{q}, 2_{\bar{q}}, 3_{Q}, 4_{\bar{Q}}, 5_{g}\right)=A^{(L)}\left(1_{q}, 2_{\bar{q}}, 3_{Q}, 4_{\bar{Q}}, 5_{g}\right)-2^{L} \mathscr{P}_{q \bar{q} Q \bar{Q}_{g}}^{(L)}(\varepsilon) A^{(0)}\left(1_{q}, 2_{\bar{q}}, 3_{Q}, 4_{\bar{Q}}, 5_{g}\right) .
$$

The colour ordered amplitude $A^{(L)}$, the finite remainder $\mathscr{F}^{(L)}$ and the pole function $\mathscr{P}_{q \bar{q} Q \bar{Q} g}^{(L)}$ can be decomposed further according to the closed fermion loop contributions

$$
\begin{aligned}
A^{(L)}\left(1_{q}, 2_{\bar{q}}, 3_{Q},{ }_{\bar{Q}}, 5_{g}\right) & =N_{c}^{L} \sum_{i=0}^{L}\left(\frac{N_{f}}{N_{c}}\right)^{i} A^{(L),[i]}\left(1_{q}, 2_{\bar{q}}, 3_{Q}, 4_{\bar{Q}}, 5_{g}\right), \\
\mathscr{F}^{(L)}\left(1_{q}, 2_{\bar{q}}, 3_{Q}, 4_{\bar{Q}}, 5_{g}\right) & =N_{c}^{L} \sum_{i=0}^{L}\left(\frac{N_{f}}{N_{c}}\right)^{i} \mathscr{F}^{(L),[i]}\left(1_{q}, 2_{\bar{q}}, 3_{Q}, 4_{\bar{Q}}, 5_{g}\right), \\
\mathscr{P}_{q \bar{q} Q \bar{Q} g}^{(L)}(\varepsilon) & =N_{c}^{L} \sum_{i=0}^{L}\left(\frac{N_{f}}{N_{c}}\right)^{i} \mathscr{P}_{q \bar{q} Q \bar{Q} g}^{(L),[i]}(\varepsilon),
\end{aligned}
$$

where $N_{f}$ is the number of light quarks circulating in the loop.

At two-loop the pole function $\mathscr{P}_{q \bar{q} Q \bar{Q} g}^{(2)}$ is given by [30-33]

$\mathscr{P}_{q \bar{q} Q \bar{Q} g}^{(2)}(\varepsilon)=I_{1}(\varepsilon)\left(I_{1}(\varepsilon)+\frac{\widehat{\mathscr{F}}^{(1)}}{2}\right)+I_{2}(\varepsilon)+\frac{5}{2} \frac{\beta_{0}}{2 \varepsilon}\left(I_{1}(\varepsilon)+\frac{\widehat{\mathscr{F}}^{(1)}}{2}+\frac{3}{2} \frac{\beta_{0}}{2 \varepsilon}\right)-\frac{3}{2}\left(\frac{5}{4} \frac{\beta_{0}^{2}}{(2 \varepsilon)^{2}}-\frac{\beta_{1}}{8 \varepsilon}\right)$,

and at one-loop by

$$
\mathscr{P}_{q \bar{q} Q \bar{Q} g}^{(1)}(\varepsilon)=I_{1}(\varepsilon)+\frac{3}{2} \frac{\beta_{0}}{2 \varepsilon}
$$

where

$$
\widehat{\mathscr{F}}^{(L)}\left(1_{q}, 2_{\bar{q}}, 3_{Q}, 4_{\bar{Q}}, 5_{g}\right)=\frac{\mathscr{F}^{(L)}\left(1_{q}, 2_{\bar{q}}, 3_{Q}, 4_{\bar{Q}}, 5_{g}\right)}{A^{(0)}\left(1_{q}, 2_{\bar{q}}, 3_{Q}, 4_{\bar{Q}}, 5_{g}\right)},
$$

is the finite remainder normalised to the tree level amplitude. To obtain the two-loop pole function $\mathscr{P}_{q \bar{q} Q \bar{Q} g}^{(2)}(\varepsilon)$, the one-loop finite remainder $\widehat{\mathscr{F}}^{(1)}$ in Eq. (4.9) must be evaluated up to $\mathscr{O}\left(\varepsilon^{2}\right)$. Note that the first two terms of Eq. (4.9) are the universal two-loop IR poles while the last two terms are the UV counterterms. We work in the t'Hooft-Veltman (HV) scheme where the spin dimension is set to $4-2 \varepsilon$.

We have derived analytic results for two-loop $q \bar{q} Q \bar{Q} g$ colour-ordered amplitudes using momentum twistor parametrisation in [2], including the $\left(N_{f} / N_{c}\right)^{0},\left(N_{f} / N_{c}\right)^{1}$ and $\left(N_{f} / N_{c}\right)^{2}$ contributions specified in Eq. (4.6), for,,+--+++-+-+-+-++ and -++-+ helicity configurations. In Table 2 we show total degree and the number of independent coefficients for each helicity configurations. We note that the complexity of the rational functions to be reconstructed depends also on the kinematic variables that are being used. The degree $c_{l}^{F, h}$ can be lowered, for example, by using $s_{i j}$ and tr $_{5}$ instead of momentum twistor variables [8]. Further improvements can also be made by identifying the denominator structures of the coefficients of pentagon functions and applying partial fractioning before performing functional reconstruction [8]. 


\begin{tabular}{|l|c|c|c|}
\hline helicity & $\left(n_{\max }, d_{\max }\right)$ & $n_{\text {points }}$ & $n_{\text {indep }}$ \\
\hline$\widehat{\mathscr{F}}_{+---++}^{(2),[0]}$ & $(26,25)$ & 23036 & 33 \\
$\widehat{\mathscr{F}}_{+-++-+}^{(2),[0]}$ & $(27,27)$ & 32473 & 45 \\
$\widehat{\mathscr{F}}_{-+++-+}^{(2),[0}$ & $(33,33)$ & 41433 & 49 \\
$\widehat{\mathscr{F}}_{-+--++}^{(2),[0}$ & $(33,33)$ & 47365 & 45 \\
\hline$\widehat{\mathscr{F}}_{+--++}^{(2),[1]}$ & $(23,22)$ & 15683 & 25 \\
$\widehat{\mathscr{F}}_{+-++-+}^{(2),[1]}$ & $(27,27)$ & 30801 & 32 \\
$\widehat{\mathscr{F}}_{-+++-+}^{(2)[1]}$ & $(27,28)$ & 22273 & 25 \\
$\widehat{\mathscr{F}}_{-+--++}^{(2),[1}$ & $(33,33)$ & 41601 & 32 \\
\hline$\widehat{\mathscr{F}}_{+--+++}^{(2),[2]}$ & $(9,9)$ & 801 & 3 \\
$\widehat{\mathscr{F}}_{+-++-+}^{(2),[2]}$ & $(11,11)$ & 1301 & 3 \\
$\widehat{\mathscr{F}}_{-+++-+}^{(2),[2]}$ & $(10,10)$ & 881 & 3 \\
$\widehat{\mathscr{F}}_{-+--++}^{(2),[2]}$ & $(9,9)$ & 705 & 3 \\
\hline
\end{tabular}

Table 2: The highest degree of polynomials in the numerator and denominator of $\bar{c}_{l^{\prime}}^{F},\left(n_{\max }, d_{\max }\right)$, the number of reconstruction points, $n_{\text {points }}$, and the number of independent coefficients, $n_{\text {indep }}$ of the two-loop finite remainder normalized to the tree level amplitude, for each independent helicity configurations.

\section{Summary}

In these proceedings we have presented a computational framework that combines Feynman diagram input, integrand reduction, numerical sampling over finite fields and analytic reconstruction techniques, that is applied to calculate two-loop five-point amplitudes in QCD. With the analytic form of planar massless five-parton amplitudes are available in the literature, efforts now has shifted toward computing the non-planar contributions in order to be able to lift the leading colour approximation, as well as looking at $2 \rightarrow 3$ processes with more scales involved, such as $H j j, V j j$ and $V V j$. The availability of analytic solutions of the master integrals has proven to be vital in deriving analytic representations of the two-loop multiscale amplitudes. The numerical evaluation of leading colour $W+4$ parton amplitudes that is presented in these proceedings, shall serve as a first step towards obtaining compact analytic representations.

\section{Acknowledgements}

We thank Simon Badger, Christian Brønnum-Hansen and Tiziano Peraro for discussions. This project has received funding from the European Union's Horizon 2020 research and innovation programme under grant agreement No 772099.

\section{References}

[1] T. Peraro JHEP 12 (2016) 030, [1608.01902]. 
[2] S. Badger, C. Brønnum-Hansen, H. B. Hartanto, and T. Peraro Phys. Rev. Lett. 120 (2018), no. 9 092001, [1712.02229].

[3] S. Abreu, F. Febres Cordero, H. Ita, B. Page, and M. Zeng Phys. Rev. D97 (2018), no. 11116014 , [1712.03946].

[4] S. Badger, C. Brønnum-Hansen, T. Gehrmann, H. B. Hartanto, J. Henn, N. A. Lo Presti, and T. Peraro PoS LL2018 (2018) 006, [1807. 0970 9].

[5] S. Abreu, F. Febres Cordero, H. Ita, B. Page, and V. Sotnikov JHEP 11 (2018) 116, [1809. 09067 ].

[6] S. Badger, C. Brønnum-Hansen, H. B. Hartanto, and T. Peraro JHEP 01 (2019) 186, [1811. 11699 ].

[7] S. Abreu, J. Dormans, F. Febres Cordero, H. Ita, and B. Page Phys. Rev. Lett. 122 (2019), no. 8 082002, [1812.04586].

[8] S. Abreu, J. Dormans, F. Febres Cordero, H. Ita, B. Page, and V. Sotnikov JHEP 05 (2019) 084, [1904.00945].

[9] S. Abreu, L. J. Dixon, E. Herrmann, B. Page, and M. Zeng Phys. Rev. Lett. 122 (2019), no. 12 121603, [1812.08941].

[10] D. Chicherin, T. Gehrmann, J. M. Henn, P. Wasser, Y. Zhang, and S. Zoia Phys. Rev. Lett. 122 (2019), no. 12 121602, [1812.11057].

[11] S. Abreu, L. J. Dixon, E. Herrmann, B. Page, and M. Zeng JHEP 03 (2019) 123, [1901. 08563 ].

[12] D. Chicherin, T. Gehrmann, J. M. Henn, P. Wasser, Y. Zhang, and S. Zoia JHEP 03 (2019) 115, [1901.05932].

[13] S. Badger, D. Chicherin, T. Gehrmann, G. Heinrich, J. M. Henn, T. Peraro, P. Wasser, Y. Zhang, and S. Zoia Phys. Rev. Lett. 123 (2019), no. 7 071601, [1905.03733].

[14] P. Nogueira J. Comput. Phys. 105 (1993) 279-289.

[15] G. Cullen, M. Koch-Janusz, and T. Reiter Comput. Phys. Commun. 182 (2011) 2368-2387, [1008.0803].

[16] A. Hodges JHEP 05 (2013) 135, [0 905 . 1473].

[17] G. Ossola, C. G. Papadopoulos, and R. Pittau Nucl. Phys. B763 (2007) 147-169, [hep-ph/0609007].

[18] R. N. Lee 1212.2685.

[19] S. Laporta Int. J. Mod. Phys. A15 (2000) 5087-5159, [hep-ph / 0102033$].$

[20] T. Peraro JHEP 07 (2019) 031, [1905.08019].

[21] T. Gehrmann and E. Remiddi Nucl. Phys. B601 (2001) 248-286, [hep-ph/ 0008287$].$

[22] T. Gehrmann, A. von Manteuffel, and L. Tancredi JHEP 09 (2015) 128, [1503. 04812 ].

[23] A. von Manteuffel and L. Tancredi JHEP 06 (2015) 197, [1503. 08835].

[24] J. M. Henn, K. Melnikov, and V. A. Smirnov JHEP 05 (2014) 090, [1 402 . 70 78 ].

[25] F. Caola, J. M. Henn, K. Melnikov, and V. A. Smirnov JHEP 09 (2014) 043, [1 404 . 5590].

[26] C. G. Papadopoulos, D. Tommasini, and C. Wever JHEP 01 (2015) 072, [1 409.6114 ].

[27] C. G. Papadopoulos, D. Tommasini, and C. Wever JHEP 04 (2016) 078, [1511. 0940 4]. 
[28] A. V. Smirnov Comput. Phys. Commun. 204 (2016) 189-199, [1511. 03614 ].

[29] S. Borowka, G. Heinrich, S. Jahn, S. P. Jones, M. Kerner, J. Schlenk, and T. Zirke Comput. Phys. Commun. 222 (2018) 313-326, [1703.09692].

[30] S. Catani Phys. Lett. B427 (1998) 161-171, [hep-ph/9802439].

[31] T. Becher and M. Neubert JHEP 06 (2009) 081, [0 903.1126 ]. [Erratum: JHEP11,024(2013)].

[32] T. Becher and M. Neubert Phys. Rev. Lett. 102 (2009) 162001, [0901. 0722]. [Erratum: Phys. Rev. Lett.111,no.19,199905(2013)].

[33] E. Gardi and L. Magnea JHEP 03 (2009) 079, [0 901 . 1091].

[34] T. Gehrmann, J. M. Henn, and N. A. Lo Presti JHEP 10 (2018) 103, [1807 . 09812 ]. 